Also available at http://amc-journal.eu

ISSN 1855-3966 (printed edn.), ISSN 1855-3974 (electronic edn.)

ARS MATHEMATICA CONTEMPORANEA 10 (2016) 223-235

\title{
Isospectral genus two graphs are isomorphic
}

\author{
Alexander Mednykh, Ilya Mednykh \\ Sobolev Institute of Mathematics, 630090, Novosibirsk, Russia \\ Novosibirsk State University, 630090, Novosibirsk, Russia \\ Siberian Federal University, 660041, Krasnoyarsk, Russia
}

Received 10 March 2013, accepted 24 July 2015, published online 3 October 2015

\begin{abstract}
By a graph we mean a finite connected multigraph without bridges. The genus of a graph is the dimension of its homology group. Two graphs are isospectral is they share the same Laplacian spectrum. We prove that two genus two graphs are isospectral if and only if they are isomorphic. Also, we present two isospectral bridgeless genus three graphs that are not isomorphic.

The paper is motivated by the following open problem posed by Peter Buser: are isospectral Riemann surfaces of genus two isometric?
\end{abstract}

Keywords: Graph, Laplacian spectrum, isospectral graphs, Laplacian polynomial, spanning tree.

Math. Subj. Class.: 05C50, 15A18, 58J53

\section{Introduction}

Over the last decade, a few discrete versions of the theory of Riemann surfaces were created $([1,18,2,8,11])$. In these theories, the role of Riemann surfaces is played by graphs. The genus of a graph is the dimension of its homology group. Under these assumptions, the theory of Jacobi manifolds is constructed and analogues of the Riemann-Hurwitz and Riemann-Roch theorems were proved. Counterparts of many other theorems from the classical theory of Riemann surfaces were derived in the discrete case $([9,10,16])$.

Since the classical paper by Mark Kac [14], the question of what geometric properties of a manifold are determined by its Laplace operator has inspired many intriguing results. One class of manifolds whose spectral theory has been studied with many beautiful results is the class of compact Riemann surfaces with the canonical constant curvature metric. Wolpert [19] showed that a generic Riemann surface is determined by its Laplace spectrum. Nevertheless, pairs of isospectral non-isometric Riemann surfaces in every genus $\geq 4$ are known. See papers by Buser [7], Brooks and Tse [5], and others. There are also examples of

E-mail addresses: smedn@mail.ru (Alexander Mednykh), ilyamednykh@mail.ru (Ilya Mednykh) 
isospectral non-isometric surfaces of genus two and three with variable curvature $([5,3])$. At the same time, isospectral genus one Riemann surfaces (flat tori) are isometric [4]. Similar results are also known for graphs $([12,13])$.

Peter Buser [6] posed an interesting problem: are two isospectral Riemann surfaces of genus two isometric? Up to our knowledge the problem is still open but, quite likely, can be solved positively. The aim of this paper is to give a positive solution of an analogous problem for bridgeless graphs of genus two (Theorem 3.1). Also, we show that there are two isospectral bridgeless graphs of genus three that are not isomorphic (Figure 5). Because of the intrinsic link between Riemann surfaces and graphs we hope that our result will be helpful to make a progress in solution of the Buser problem.

\section{Preliminary results}

\subsection{Laplacian matrix and Laplacian spectrum}

The Laplacian matrix of a graph and its eigenvalues can be used in several areas of mathematical research and have a physical interpretation in various physical and chemical theories. The related adjacency matrix of a graph and its eigenvalues were much more investigated in the past than the Laplacian matrix. At the same time, the Laplacian spectrum is much more natural and more important than the adjacency matrix spectrum because of it numerous application in mathematical physics, chemistry and financial mathematics.

Graphs in this paper are finite and undirected, but they may have loops and multiple edges. Denote by $V(G)$ and $E(G)$, respectively, the number of vertices and edges of a graph $G$. Following [2] we denote by $g(G)=E(G)-V(G)+1$ the genus of $G$. This is the dimension of the first homology group of $G$. In graph theory, the term "genus" is traditionally used for a different concept, namely, the smallest genus of any surface in which the graph can be embedded, and the integer $g=g(G)$ is called the cyclomatic or the Betti number of $G$. We call $g$ the genus of $G$ in order to highlight the analogy with Riemann surfaces.

A bridge is an edge of a graph $G$ whose deletion increases the number of connected components. Equivalently, an edge is a bridge if and only if it is not contained in any cycle. A graph is said to be bridgeless if it contains no bridges.

Let $G$ be a graph. Denote by $\mathcal{V}(G)$ and $\mathcal{E}(G)$ the set of vertices and edges of a graph $G$ respectively. For each $u, v \in \mathcal{V}(G)$, we set $a_{u v}$ to be equal to the number of edges between $u$ and $v$. The matrix $A=A(G)=\left[a_{u v}\right]_{u, v \in \mathcal{V}(G)}$, is called the adjacency matrix of the graph $G$.

Let $d(v)$ denote the valency of $v \in \mathcal{V}(G), d(v)=\sum_{u} a_{u v}$, and let $D=D(G)$ be the diagonal matrix indexed by $\mathcal{V}(G)$ and with $d_{v v}=d(v)$. The matrix $L=L(G)=$ $D(G)-A(G)$ is called the Laplacian matrix of $G$. It should be noted that loops have no influence on $L(G)$. Throughout the paper we shall denote by $\mu(G, x)$ the characteristic polynomial of $L(G)$. For brevity, we will call $\mu(G, x)$ the Laplacian polynomial of $G$. Its roots will be called the Laplacian eigenvalues (or sometimes just eigenvalues) of $G$. They will be denoted by $\mu_{1}(G) \leq \mu_{2}(G) \leq \ldots \leq \mu_{n}(G),(n=V(G))$, always enumerated in increasing order and repeated according to their multiplicity. Recall [17] that for connected graph $G$ we always have $\mu_{1}(G)=0$ and $\mu_{2}(G)>0$.

Two graphs $G$ and $H$ are called Laplacian isospectral (or isospectral) if their Laplacian polynomials coincide: $\mu(G, x)=\mu(H, x)$.

The matrix $L(G)$ is sometimes called the Kirchhoff matrix of $G$ due to its role in the 
well-known Matrix-Tree Theorem which is usually attributed to Kirchhoff. A generalization of the Matrix-Tree-Theorem was obtained in 1967 by A. K. Kel'mans who gave a combinatorial interpretation to all the coefficients of $\mu(X, x)$ in terms of the numbers of certain subforests of a graph $X$; see [15] and [17] for references and history of question. We present the result by Kel'mans in the following form.

Theorem 2.1. [15] If $\mu(X, x)=x^{n}-c_{1} x^{n-1}+\ldots+(-1)^{i} c_{i} x^{n-i}+\ldots+(-1)^{n-1} c_{n-1} x$ then

$$
c_{i}=\sum_{S \subset V,|S|=n-i} T\left(X_{S}\right),
$$

where $T(H)$ is the number of spanning trees of $H$, and $X_{S}$ is obtained from $X$ by identifying all points of $S$ to a single point.

\subsection{Theta graphs}

Let $u$ and $v$ are two (not necessary distinct) vertices. Denote by $\Theta(k, l, m)$ the graph consisting of three internally disjoint paths joining $u$ to $v$ with lengths $k, l, m \geq 0$ (see Fig. $1)$. We set $\sigma_{1}=\sigma_{1}(k, l, m)=k+l+m, \sigma_{2}=\sigma_{2}(k, l, m)=k l+l m+k m$, and $\sigma_{3}=\sigma_{3}(k, l, m)=k l m$. It is easy to see that two graphs $\Theta(k, l, m)$ and $\Theta\left(k^{\prime}, l^{\prime}, m^{\prime}\right)$ are isomorphic if and only if the unordered triples $\{k, l, m\}$ and $\left\{k^{\prime}, l^{\prime}, m^{\prime}\right\}$ coincide; equivalently, $\sigma_{1}=\sigma_{1}^{\prime}, \sigma_{2}=\sigma_{2}^{\prime}$ and $\sigma_{3}=\sigma_{3}^{\prime}$, where $\sigma_{1}^{\prime}=\sigma_{1}\left(k^{\prime}, l^{\prime}, m^{\prime}\right), \sigma_{2}^{\prime}=\sigma_{2}\left(k^{\prime}, l^{\prime}, m^{\prime}\right)$, and $\sigma_{3}^{\prime}=\sigma_{1}\left(k^{\prime}, l^{\prime}, m^{\prime}\right)$.
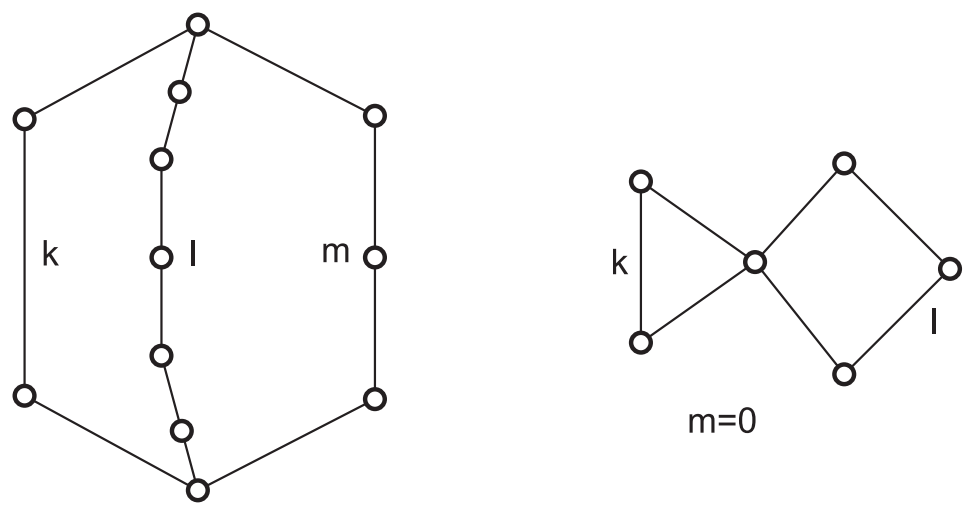

Figure 1: Theta graph $\Theta(k, l, m)$.

We make the following useful observations:

(i) If $\sigma_{2}>0$, then $\Theta(k, l, m)$ is a graph of genus two. In this case at least two of numbers $\{k, l, m\}$ are positive.

(ii) If $\sigma_{1}>0, \sigma_{2}=0$, then $\Theta(k, l, m)$ is a graph of genus one. Then exactly one of numbers $\{k, l, m\}$ is positive and the other two are zero. Moreover, $\Theta(k, l, m)=$ $C_{k+l+m}$ is a cyclic graph with $k+l+m$ edges. 
(iii) If $\sigma_{1}=0$, then $k=l=m=0$ and $\Theta(k, l, m)$ is a graph of genus zero. More precisely, $\Theta(k, l, m)=\Theta(0,0,0)$ consists of one vertex.

Lemma 2.2. Let $G$ be an arbitrary bridgeless graph of genus two. Then $G$ is isomorphic to $\Theta(k, l, m)$ for some $k, l, m$ with $\sigma_{2}=k l+l m+k m>0$.

Proof. Since the graph $G$ is bridgeless it has no vertices of valency one. Denote by $H$ the graph obtained from $G$ by deleting of all vertices of valency two. Suppose that $H$ has $V$ vertices of valences $n_{1}, n_{2}, \ldots, n_{V}$ and $E$ edges. Since the valency of each vertex of $H$ is at least three we have $n_{i} \geq 3, i=1,2, \ldots, V$. Note that deleting of a vertex of valency two decreases the number of vertices and the number of edges of a graph by one. So, it does not affect the genus and $H$ is still a graph of genus two. Thus $g(H)=1-V+E=2$ and $E=V+1$. Counting the sum of valences of $H$ through vertices and through edges we obtain

$$
n_{1}+n_{2}+\ldots+n_{V}=2 E
$$

Hence

$$
3 V \leq n_{1}+n_{2}+\ldots+n_{V}=2 E=2 V+2
$$

or $V \leq 2$.

If $V=1$ then $n_{1}=4$ and $H$ is the figure eight graph consisting of one vertex and two loops. Putting back the vertices of valency two on the graph $H$ we obtain the graph $G$ isomorphic to $\Theta(k, l, 0)$ for some positive $k$ and $l$. In particular, $\sigma_{2}=k l>0$.

If $V=2$ then $n_{1}=n_{2}=3$ and $H$ is the theta graph consisting of two vertices and three edges. The graph $G$ is obtained from $H$ by adding the vertices of valency two. Hence, $G$ is isomorphic to $\Theta(k, l, m)$ for some positive $k, l, m$.

\section{Main results}

\subsection{The main theorem and lemmas}

The main result of the paper is the following theorem.

Theorem 3.1. Two genus two bridgeless graphs are Laplacian isospectral if and only if they are isomorphic.

The proof of the theorem is based on the following three lemmas.

Lemma 3.2. Let $G=\Theta(k, l, m)$ be a theta graph and let $\mu(G, x)=x^{n}-c_{1} x^{n-1}+\ldots+$ $(-1)^{n-1} c_{n-1} x$ be its Laplacian polynomial. Then $n=k+l+m-1, c_{1}=2(k+l+m)$ and $c_{n-1}=(k l+l m+k m)(k+l+m-1)$.

Proof. The number of vertices, edges and spanning trees of graph $G$ are given by

$$
V(G)=k+l+m-1, E(G)=k+l+m, T(G)=k l+l m+k m .
$$

Then by ([15], formulas 2.15 and 2.16) we have $n=V(G)=k+l+m-1, c_{1}=$ $2 E(G)=2(k+l+m)$ and $c_{n-1}=V(G) \cdot T(G)=(k l+l m+k m)(k+l+m-1)$. 
Lemma 3.3. Let $G=\Theta(k, l, m)$ be a theta graph and let $\mu(G, x)=x^{n}-c_{1} x^{n-1}+\ldots+$ $(-1)^{n-1} c_{n-1} x$ be its Laplacian polynomial. Then

$$
c_{n-2}=A\left(\sigma_{1}, \sigma_{2}\right)+B\left(\sigma_{1}, \sigma_{2}\right) \sigma_{3}
$$

where $A(s, t)=\left(4 t-3 s t-2 s^{2} t+s^{3} t+4 t^{2}-s t^{2}\right) / 12, B(s, t)=\left(3-4 s+s^{2}-3 t\right) / 12$, $\sigma_{1}=k+l+m, \sigma_{2}=k l+l m+k m$, and $\sigma_{3}=k l m$.

Proof. By Theorem 2.1

$$
c_{n-2}=\sum_{S \subset V,|S|=2} T\left(X_{S}\right),
$$

where $X_{S}$ runs through all graphs obtained from $G=\Theta(k, l, m)$ by gluing two vertices. There are exactly four types of such graphs $G_{1}, G_{2}, G_{3}$, and $G_{4}$ shown in the Fig. 2. We will enumerate the spanning trees of each type separately.

Type $G_{1}$. Glue two 3 -valent vertices of graph $G$. As a result we obtain the graph $G_{1}$ shown on Fig. 2. The number of spanning trees of this graph is $T_{1}=T\left(C_{k}\right) \cdot T\left(C_{l}\right)$. $T\left(C_{m}\right)=k l m$.

Type $G_{2}$. Glue one 3 -valent and one 2-valent vertices of graph $G$. The graph of type $G_{2}$ shown in Fig. 2 is obtained by gluing the upper 3-valent of graph $G$ and a 2 -valent vertex on the path of $G$ labelled by $k$. For given $i, 1 \leq i \leq k-1$ the number of spanning trees for a graph of type $G_{2}$ is equal to $T\left(C_{i}\right) \cdot T(\Theta(k-i, l, m))=i \sigma_{2}(k-i, l, m)$. We set $F(k, l, m)=\sum_{i=1}^{k-1} i \sigma_{2}(k-i, l, m)$. Then the total number of spanning trees for graphs of type $G_{2}$ is

$$
T_{2}=2(F(k, l, m)+F(l, m, k)+F(m, k, l)) .
$$

The multiple 2 is needed since the graph $\Theta(k, l, m)$ has two 3-valent vertices.

Type $G_{3}$. Glue two 2 -valent vertices of graph $G$ lying on different paths. We choose one of them on the path labelled by $k$ and the second on the path labbeled by $l$. Fix $i, 1 \leq$ $i \leq k-1$ and $j, 1 \leq j \leq l-1$ and consider a graph of type $G_{3}$ shown in Fig. 2. This is a graph of genus three. To create a spanning tree on this graph we have to delete three edges. There are two different ways to do this. Firstly, we delete edges on three of the four paths labeled by $i, j, k-i$ and $l-j$. This be done in $\sigma_{3}(i, j, k-i, l-j)$ ways, where $\sigma_{3}(x, y, z, t)=x y z+x y t+x z t+y z t$. Secondly, if we delete an edge from the path labeled by $m$ (in $m$ possible ways) then we have to remove one edge from the pair of paths $i, j$ and one edge from the pair $k-i, k-j$. Then we have $m((i+j)(k-i+l-j))$ possibilities to obtain a tree. As the result graph under consideration has $G_{3}(i, j, k, l, m)=\sigma_{3}(i, j, k-$ $i, l-j)+m((i+j)(k-i+l-j)$ spanning trees. We set

$$
J(k, l, m)=\sum_{i=1}^{k-1} \sum_{j=1}^{l-1} G_{3}(i, j, k, l, m) .
$$

Then the total number of spanning trees for graphs of type $G_{3}$ is

$$
T_{3}=J(k, l, m)+J(l, m, k)+J(m, k, l) .
$$

Type $G_{4}$. Glue two 2 -valent vertices lying on the same path of graph $G$. Choose the path labelled by $k$. Let us fix $i$ and $j$ such that $1 \leq i<j \leq k-1$. Then the number 
of spanning trees for a given graph of type $G_{4}$ is $T\left(C_{j-i}\right) T(\Theta(k+i-j, l, m))=(j-$ i) $\sigma_{2}(k+i-j, l, m)$. We set

$$
H(k, l, m)=\sum_{i=1}^{k-2} \sum_{j=i+1}^{k-1}(j-i) \sigma_{2}(k+i-j, l, m) .
$$

As a result, the number of spanning trees of the given type is

$$
T_{4}=H(k, l, m)+H(l, m, k)+H(m, k, l) .
$$

Putting the obtained formulas in Mathematica 8 by (3.1) we get

$$
c_{n-2}=T_{1}+T_{2}+T_{3}+T_{4}=A\left(\sigma_{1}, \sigma_{2}\right)+B\left(\sigma_{1}, \sigma_{2}\right) \sigma_{3} .
$$



$\mathrm{G}_{1}$

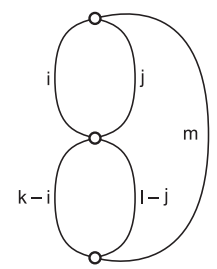

$\mathrm{G}_{3}$



$\mathrm{G}_{2}$

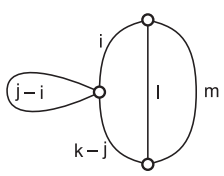

$\mathrm{G}_{4}$

Figure 2: The graphs obtained from $\Theta(k, l, m)$ by gluing two vertices

Lemma 3.4. Let $G=\Theta(k, l, m)$ be a theta graph and let

$$
\mu(G, x)=x^{n}-c_{1} x^{n-1}+\ldots+(-1)^{n-1} c_{n-1} x
$$

be its Laplacian polynomial. Then

$$
c_{n-3}=C\left(\sigma_{1}, \sigma_{2}\right)+D\left(\sigma_{1}, \sigma_{2}\right) \sigma_{3}+E\left(\sigma_{1}, \sigma_{2}\right) \sigma_{3}^{2},
$$

where

$$
\begin{aligned}
& C(s, t)=\left(-34 t+21 s t+25 s^{2} t-10 s^{3} t-3 s^{4} t+s^{5} t-50 t^{2}+10 s t^{2}\right. \\
&\left.+12 s^{2} t^{2}-2 s^{3} t^{2}-16 t^{3}+s t^{3}\right) / 360, \\
& D(s, t)=\left(-45+50 s+5 s^{2}-12 s^{3}+2 s^{4}+24 s t-9 s^{2} t+15 t^{2}\right) / 360, \\
& E(s, t)=-3(-8+3 s) / 360 .
\end{aligned}
$$


Proof. By Theorem 2.1

$$
c_{n-3}=\sum_{S \subset V,|S|=3} T\left(X_{S}\right),
$$

where $X_{S}$ runs through all graphs obtained from $G=\Theta(k, l, m)$ by gluing three vertices. There are six types of such graphs $W_{1}, W_{2}, W_{3}, W_{4}, W_{5}$, and $W_{6}$ shown on the Fig. 3 . We examine the spanning trees of each type separately.

Type $W_{1}$. To create a graph of type $W_{1}$ we identify two 3 -valent vertices of graph $G$ and one 2 -valent vertex of $G$ (say on the path labelled by $k$ ). The obtained graph is shown in the Fig. 3, has $i(k-i) l m$ spanning trees. Consider the sum $F^{w}(k, l, m)=\sum_{i=1}^{k-1} i(k-i) l m$. Find the total number of spanning trees for graphs of type $W_{1}$ by the formula

$$
T_{1}^{w}=F^{w}(k, l, m)+F^{w}(l, m, k)+F^{w}(m, k, l) .
$$

Type $W_{2}$. Glue one 3 -valent vertices of graph $G$ and two 2-valent vertices lying on different paths of $G$ (say on the paths labelled by $k$ an $l$ ), obtaining a graph in Fig. 3. For given $i$ and $j, 1 \leq i \leq k-1,1 \leq j \leq l-1$, the number of spanning trees for graph of type $W_{2}$ is $i j \sigma_{2}(k-i, l-j, m)$. We set $H^{w}(k, l, m)=\sum_{i=1}^{k-1} \sum_{j=1}^{l-1} i j \sigma_{2}(k-i, l-j, m)$. Taking into account that graph $\Theta(k, l, m)$ has two 3 -valent vertices we obtain the following formula the number of spanning trees for graphs of type $W_{2}$ :

$$
T_{2}^{w}=2\left(H^{w}(k, l, m)+H^{w}(l, m, k)+H^{w}(m, k, l)\right) .
$$

Type $W_{3}$. Glue one 3 -valent vertices and two 2-valent vertices lying on the same path of $G$. For fixed $i$ and $j, 1 \leq i<j \leq k-1$, we have $i(j-i) \sigma_{2}(k-j, l, m)$ spanning trees for graph of type $W_{3}$. Summing over $i$ and $j$ we get

$$
J^{w}(k, l, m)=\sum_{i=1}^{k-2} \sum_{j=i+1}^{k-1} i(j-i) \sigma_{2}(k-j, l, m) .
$$

Finally, the number of spanning trees for graphs of type $W_{3}$ is given by

$$
T_{3}^{w}=2\left(J^{w}(k, l, m)+J^{w}(l, m, k)+J^{w}(m, k, l)\right) .
$$

Type $W_{4}$. Glue three 2 -valent vertices all lying on different paths of $G$. Fix $i, j$ and $s, 1 \leq i \leq k-1,1 \leq j \leq l-1,1 \leq s \leq m-1$. Then the number of spanning trees for a given graph of type $W_{4}$ is equal to $\sigma_{2}(i, j, s) \sigma_{2}(k-i, l-j, m-s)$. Summing over $i, j$ and $s$ we obtain the total number of spanning trees for graphs of type $W_{4}$ :

$$
T_{4}^{w}=\sum_{i=1}^{k-1} \sum_{j=1}^{l-1} \sum_{s=1}^{m-1} \sigma_{2}(i, j, s) \sigma_{2}(k-i, l-j, m-s) .
$$

Type $W_{5}$. Glue two 2-valent vertices lying on a path and one 2-valent vertex lying on the other path of $G$. Denote by $G_{3}(i, j, k, l, m)$ the graph of type $G_{3}$ shown in Fig. 2. From the proof of previous Lemma we have $T\left(G_{3}(i, j, k, l, m)\right)=\sigma_{3}(i, j, k-i, l-j)+m((i+$ 
$j)(k-i+l-j))$. Fix $i, j$ and $s, 1 \leq i<j \leq k-1,1 \leq s \leq l-1$. Then the number of spanning trees for a graph of type $W_{5}$ in Fig. 3 is equal to

$$
T\left(C_{j-i}\right) T\left(G_{3}(i, s, k+i-j, l, m)\right)=(j-i) T\left(G_{3}(i, s, k+i-j, l, m)\right) .
$$

Consider the sum

$$
K^{w}(k, l, m)=\sum_{i=1}^{k-2} \sum_{j=i+1}^{k-1} \sum_{s=1}^{l-1}(j-i) T\left(G_{3}(i, s, k+i-j, l, m)\right) .
$$

Then the number of spanning trees for graphs of type $W_{3}$ is given by

$$
\begin{aligned}
T_{5}^{w}=K^{w}(k, l, m)+K^{w}(l, & m, k)+K^{w}(m, k, l) \\
& +K^{w}(k, m, l)+K^{w}(l, k, m)+K^{w}(m, l, k) .
\end{aligned}
$$

Type $W_{6}$. Glue three 2 -valent vertices on the same path of $G$. Fixed $i, j$ and $s$ such that $1 \leq s<i<j \leq k-1$. Then the number of spanning trees for a given graph of type $W_{6}$ is equal to

$$
T\left(C_{i-s}\right) T\left(C_{j-i}\right) T(\Theta(k-j+s, l, m))=(i-s)(j-i) \sigma_{2}(k-j+s, l, m) .
$$

Summing over $i, j$ and $s$ we obtain

$$
L^{w}(k, l, m)=\sum_{1 \leq s<i<j \leq k-1}(i-s)(j-i) \sigma_{2}(k-j+s, l, m) .
$$

The total number of spanning trees in this case

$$
T_{6}^{w}=L^{w}(k, l, m)+L^{w}(l, m, k)+L^{w}(m, k, l) .
$$

By (3.4) and straightforward calculation in Mathematica. 8 we obtain

$$
\begin{aligned}
c_{n-3} & =T_{1}^{w}+T_{2}^{w}+T_{3}^{w}+T_{4}^{w}+T_{5}^{w}+T_{6}^{w} \\
& =C\left(\sigma_{1}, \sigma_{2}\right)+D\left(\sigma_{1}, \sigma_{2}\right) \sigma_{3}+E\left(\sigma_{1}, \sigma_{2}\right) \sigma_{3}^{2} .
\end{aligned}
$$

\subsection{Proof of the main theorem}

Proof. Let $G$ and $G^{\prime}$ be two bridgeless graphs of genus two. Then by Lemma 1 for suitable $\{k, l, m\}$ and $\left\{k^{\prime}, l^{\prime}, m^{\prime}\right\}$ we have

$$
G=\Theta(k, l, m) \text { and } G^{\prime}=\Theta\left(k^{\prime}, l^{\prime}, m^{\prime}\right) .
$$

Denote by $\mu(G, x)=x^{n}-c_{1} x^{n-1}+\ldots+(-1)^{n-1} c_{n-1} x$ and

$$
\mu\left(G^{\prime}, x\right)=x^{n^{\prime}}-c_{1} x^{n^{\prime}-1}+\ldots+(-1)^{n^{\prime}-1} c_{n^{\prime}-1} x
$$

their Laplacian polynomials. 

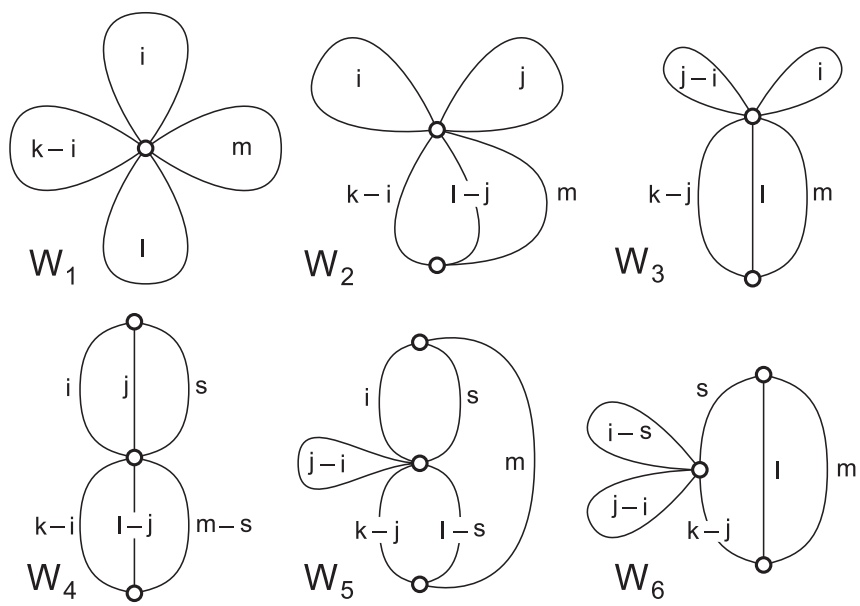

Figure 3: The graphs obtained from $\Theta(k, l, m)$ by gluing three vertices

Suppose that the graphs $G$ and $G^{\prime}$ are isospectral. Then $n^{\prime}=n, c_{1}^{\prime}=c_{1}, \ldots, c_{n-1}^{\prime}=$ $c_{n-1}$. From the second and the last equalities by Lemma 2 we obtain

$$
2 \sigma_{1}=2 \sigma_{1}^{\prime} \text { and } \sigma_{2}\left(\sigma_{1}-1\right)=\sigma_{2}^{\prime}\left(\sigma_{1}^{\prime}-1\right) .
$$

Since both graphs are of genus 2 we have $\sigma_{1}>1$ and $\sigma_{1}^{\prime}>1$. Then the system of equations (3.4) gives $\sigma_{1}=\sigma_{1}^{\prime}$ and $\sigma_{2}=\sigma_{2}^{\prime}$. The theorem will be proved if we show that $\sigma_{3}=\sigma_{3}^{\prime}$. We will do this in two steps. First of all, we note that by [13] isospectral graphs with $n \leq 5$ vertices are isomorphic. So, we can assume that $n=k+l+m-1>5$, that is, $\sigma_{1}=k+l+m>6$.

By Lemma 3,

$$
c_{n-2}=A\left(\sigma_{1}, \sigma_{2}\right)+B\left(\sigma_{1}, \sigma_{2}\right) \sigma_{3},
$$

where $A(s, t)=\left(4 t-3 s t-2 s^{2} t+s^{3} t+4 t^{2}-s t^{2}\right) / 12$ and $B(s, t)=\left(3-4 s+s^{2}-3 t\right) / 12$. obtain

Step 1. $B\left(\sigma_{1}, \sigma_{2}\right) \neq 0$. Since $c_{n-2}^{\prime}=c_{n-2}, \sigma_{1}=\sigma_{1}^{\prime}$ and $\sigma_{2}=\sigma_{2}^{\prime}$ from (3.5) we

$$
B\left(\sigma_{1}, \sigma_{2}\right) \sigma_{3}^{\prime}=B\left(\sigma_{1}, \sigma_{2}\right) \sigma_{3} .
$$

Hence $\sigma_{3}=\sigma_{3}^{\prime}$ and the theorem is proved.

Step 2. $B\left(\sigma_{1}, \sigma_{2}\right)=0$. Then by Lemma 3

$$
c_{n-3}=C\left(\sigma_{1}, \sigma_{2}\right)+D\left(\sigma_{1}, \sigma_{2}\right) \sigma_{3}+E\left(\sigma_{1}, \sigma_{2}\right) \sigma_{3}^{2},
$$

where

$$
\begin{aligned}
& C(s, t)=\left(-34 t+21 s t+25 s^{2} t-10 s^{3} t-3 s^{4} t+s^{5} t-50 t^{2}+10 s t^{2}\right. \\
&\left.+12 s^{2} t^{2}-2 s^{3} t^{2}-16 t^{3}+s t^{3}\right) / 360 \\
& D(s, t)=\left(-45+50 s+5 s^{2}-12 s^{3}+2 s^{4}+24 s t-9 s^{2} t+15 t^{2}\right) / 360 \\
& E(s, t)=-3(-8+3 s) / 360 .
\end{aligned}
$$


Since $c_{n-3}^{\prime}=c_{n-3}, \sigma_{1}=\sigma_{1}^{\prime}$ and $\sigma_{2}=\sigma_{2}^{\prime}$ from (3.7) we obtain

$$
D\left(\sigma_{1}, \sigma_{2}\right) \sigma_{3}^{\prime}+E\left(\sigma_{1}, \sigma_{2}\right){\sigma_{3}^{\prime}}^{2}=D\left(\sigma_{1}, \sigma_{2}\right) \sigma_{3}+E\left(\sigma_{1}, \sigma_{2}\right) \sigma_{3}^{2} .
$$

We note that $E\left(\sigma_{1}, \sigma_{2}\right) \neq 0$ for any integer $\sigma_{1}$. Then the above equation has two solutions with respect to $\sigma_{3}^{\prime}$. The first solution is $\sigma_{3}^{\prime}=\sigma_{3}$ and the second one is

$$
\sigma_{3}^{\prime}=-\frac{D\left(\sigma_{1}, \sigma_{2}\right)}{E\left(\sigma_{1}, \sigma_{2}\right)}-\sigma_{3}
$$

In the first case the theorem is proved. So we assume that $\sigma_{3}^{\prime}$ is given by equation (3.9). Recall that $B\left(\sigma_{1}, \sigma_{2}\right)=0$. Then $\sigma_{2}=\left(3-4 \sigma_{1}+\sigma_{1}^{2}\right) / 3$ and equation (3.9) can be rewritten in the form

$$
\sigma_{3}^{\prime}=\frac{1}{729}\left(2\left(425-357 \sigma_{1}-144 \sigma_{1}^{2}+27 \sigma_{1}^{3}\right)-\frac{490}{-8+3 \sigma_{1}}\right)-\sigma_{3} .
$$

Since $\sigma_{3}$ and $\sigma_{3}^{\prime}$ are integers the number

$$
N=2\left(425-357 \sigma_{1}-144 \sigma_{1}^{2}+27 \sigma_{1}^{3}\right)-\frac{490}{-8+3 \sigma_{1}}
$$

is an integer divisible by 729 . Moreover, $-8+3 \sigma_{1}$ is a divisor of 490 and the number $\sigma_{2}=\left(3-4 \sigma_{1}+\sigma_{1}^{2}\right) / 3$ is a positive integer. There are a finite number possibilities of a positive integer $\sigma_{1}$ to satisfy these three conditions, namely, $\sigma_{1} \in\{6,19,166\}$. The case $\sigma_{1}=6$ can be excluded since we suggested that $\sigma_{1}>6$. Another way to exclude $\sigma_{1}=6$ is to check that in this case $\sigma_{3}^{\prime}=-3-\sigma_{3}$ is negative.

Consider the remaining cases $\sigma_{1}=19$ and $\sigma_{1}=166$. By (3.10) in these cases we have $\sigma_{3}^{\prime}=348-\sigma_{3}$ and $\sigma_{3}^{\prime}=327789-\sigma_{3}$ respectively. The respective values of $\sigma_{2}$ are 96 and 8965.

Let $\sigma_{1}=19$. We have the following system of equations to find positive integer parameters $k, l, m, \sigma_{3}$ of the graph $G=\Theta(k, l, m)$ :

$$
k+l+m=19, k l+l m+m k=96, k l m=\sigma_{3} .
$$

This system has only one solution $\{k, l, m\}=\{3,4,12\}, \sigma_{3}=144$.

Now we are able to find parameters $k^{\prime}, l^{\prime}, m^{\prime}, \sigma_{3}^{\prime}$ of the graph $G^{\prime}=\Theta\left(k^{\prime}, l^{\prime}, m^{\prime}\right)$. First of all, $\sigma_{3}^{\prime}=348-\sigma_{3}=204$. Then we have

$$
k^{\prime}+l^{\prime}+m^{\prime}=19, k^{\prime} l^{\prime}+l^{\prime} m^{\prime}+m^{\prime} k=96, k^{\prime} l^{\prime} m^{\prime}=204 .
$$

The latter system has no integer solutions. So the case $\sigma_{1}=19$ is impossible.

Let $\sigma_{1}=166$. We have the following system $k, l, m, \sigma_{3}$.

$$
k+l+m=166, k l+l m+m k=8965, k l m=\sigma_{3} .
$$

This system has only one solution $\{k, l, m\}=\{39,59,68\}, \sigma_{3}=39 \cdot 59 \cdot 68$.

Find parameters $k^{\prime}, l^{\prime}, m^{\prime}, \sigma_{3}^{\prime}$ of the graph $G^{\prime}=\Theta\left(k^{\prime}, l^{\prime}, m^{\prime}\right)$. Now, $\sigma_{3}^{\prime}=327789-$ $\sigma_{3}=171321$. Then we have

$$
k^{\prime}+l^{\prime}+m^{\prime}=166, k^{\prime} l^{\prime}+l^{\prime} m^{\prime}+m^{\prime} k^{\prime}=8965, k^{\prime} l^{\prime} m^{\prime}=171321 .
$$

The system has no integer solutions. The case $\sigma_{1}=166$ is also impossible.

This completes the proof. 


\section{Final remarks}

1. The main Theorem 3.1 is not valid for genus two graphs with bridges. Indeed, the following two graphs (see Fig. 4) constructed in [12] are isospectral. They share the Laplacian polynomial

$$
-72 x+192 x^{2}-176 x^{3}+73 x^{4}-14 x^{5}+x^{6} .
$$

The first of these graphs is bridgeless, while the second one has a bridge.
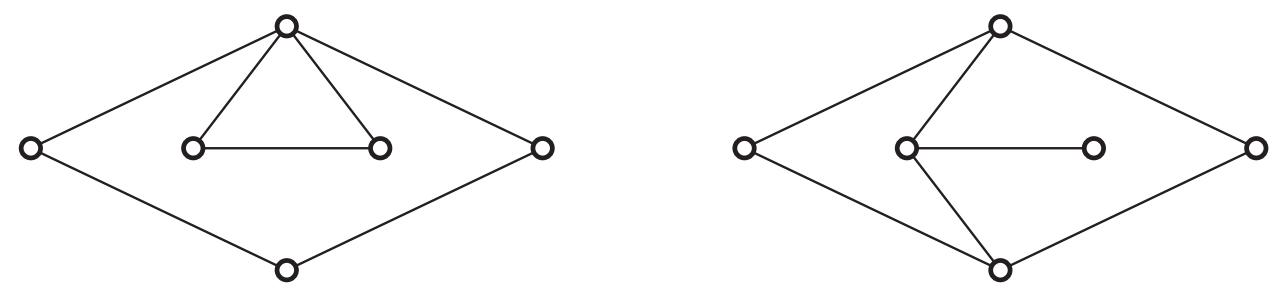

Figure 4: Isospectral graphs of genus two. The second graph has a bridge.

2. There are isospectral bridgeless graphs of genus three which are not isomorphic (see Fig. 5). These two graphs were constructed in [13].They share the Laplacian polynomial

$$
-384 x+1520 x^{2}-2288 x^{3}+1715 x^{4}-708 x^{5}+164 x^{6}-20 x^{7}+x^{8} .
$$


Figure 5: Isospectral graphs of genus three.

3. Any bridgeless graph of genus one is isomorphic to a cyclic graph $C_{n}$ for some $n \geq$ 1. If two cyclic graphs $C_{m}$ and $C_{n}$ are isospectral then their Laplace polynomials are of the same degree $m=n$. Hence, the graphs are isomorphic. 
At the same time, there are isospectral unicycle graphs [20]. For example, the two genus one graphs shown on Fig. 6 share the Laplacian polynomial

$$
28 x-146 x^{2}+250 x^{3}-194 x^{4}+75 x^{5}-14 x^{6}+x^{7} .
$$



Figure 6: Isospectral graphs of genus one.

4. One can hear the genus of a graph. That is, the genus of a graph $G$ is completely determined by its Laplace spectrum. Indeed, $g(G)=1-V(G)+E(G)$. Let $\mu(G, x)=x^{n}-c_{1} x^{n-1}+\ldots+(-1)^{n-1} c_{n-1} x$ be the Laplacian polynomial of $G$. By the arguments from the proof of Lemma 3.2 we have $n=V(G)$ and $c_{1}=2 E(G)$. Thus $V(G)$ and $E(G)$, as well as the genus, are uniquely determined by the Laplacian polynomial.

It follows from this observation, the previous remark, and the main result of the paper that the bridgeless graphs of genera one and two are recognisable by their Laplacian spectra among all bridgeless graphs.

5. One cannot hear a bridge of a graph. Indeed, the two graphs in Fig. 4 are isospectral. We are not able to recognise the existence of a bridge of the second graph by its spectrum.

\section{Acknowledgments}

The authors are thankful to the following grants for partial support of this investigation: the Russian Foundation for Basic Research (project no. 15-01-07906), the Grant of the Russian Federation Government at Siberian Federal University (grant 14.Y26.31.0006), the Program "Leading Scientific Schools" (project no. NSh-921.2012.1), the Dynasty Foundation and the Project: Mobility - enhancing research, science and education at the Matej Bel University, ITMS code: 26110230082, under the Operational Program Education cofinanced by the European Social Fund and by Slovenian-Russian grant (2014-2015). 


\section{References}

[1] R. Bacher, P. de la Harpe, and T. Nagnibeda, The lattice of integral flows and the lattice of integral cuts on a finite graph, Bull. Soc. Math. France, 125 (1997), 167-198.

[2] M. Baker, S. Norine, Harmonic morphisms and hyperelliptic graphs, Int. Math. Res. Notes, 15 (2009), 2914-2955.

[3] D. Barden and Hyunsuk Kang, Isospectral surfaces of genus two and three, Math. Proc. Cambridge Philos. Soc., 153(1) (2012), 99-110.

[4] R. Brooks, Constructing Isospectral Manifolds, Amer. Math. Monthly, 95 (9) (1988), 823-839,

[5] R. Brooks and R. Tse, Isospectral surfaces of small genus, Nagoya Math. J., 107 (1987), 13-24.

[6] P. Buser, Geometry and spectra of compact Riemann surfaces, Progress in Mathematics, 106 Birkhhäuser, Boston, MA, 1992.

[7] P. Buser, Isospectral Riemann surfaces, Ann. Inst. Fourier, 36 (1986), 167-192.

[8] L. Caporaso, Algebraic and tropical curves: comparing their moduli spaces,in: G. Farkas, I. Morrison (eds.), Handbook of Moduli, I, Advance Lectures in Mathematics,XXIV(2011),119160.

[9] L. Caporaso, Gonality of algebraic curves and graphs, in: Algebraic and Complex geometry: in honor of Klaus Hulek's 60th Birthday, Springer Procedings in Mathematics \& Statistic 71 (2014), 77-109.

[10] S. Corry, Genus bounds for harmonic group actions on finite graphs, Inter. Math. Res. Not., 19 (2011), 4515-4533.

[11] S. Corry, Harmonic Galois theory for finite graphs, H. Nakamura, F. Pop, L. Schneps, A. Tamagawa (eds.), in: Galois-Teichmüller Theory and Arithmetic Geometry, Advanced Studies in Pure Mathematics, 63 (2012), 121-140.

[12] E. R. van Dam and W. H. Haemers, Which graphs are determined by their spectrum?, Linear Algebra and its Applications, 373 (2003), 241-272.

[13] W. H. Haemers and E. Spence, Enumeration of cospectral graphs, European J. of Combin. 25(2) (2004), 199-211.

[14] M. Kac, Can one hear the shape of a drum?, Amer. Math. Monthly, 73 (4 (part II)) (1966), $1-23$.

[15] A. K. Kel'mans and V. M. Chelnokov, A certain polynomial of a graph and graphs with an extremal number of trees, J. Combin. Theory, Ser. B, 16(3) (1974), 197-214.

[16] I. A. Mednykh, On the Farkas and Accola Theorems for Graphs, Doklady Mathematics, 87(1) (2013), 65-68.

[17] B. Mohar, The Laplacian spectrum of graphs, in: Y. Alavi, G. Chartrand, O. R. Oellermann, A. J. Schwenk (eds.), Graph theory, combinatorics, and applications, Wiley, 2 (1991), 871898.

[18] H. Urakawa, A discrete analogue of the harmonic morphism and Green kernel comparison theorems, Glasgow Math. J., 42(3) (2000), 319-334.

[19] S. Wolpert, The length spectra as moduli for compact Riemann surfaces, Ann. Math. (2), 109(2) (1979), 323-351.

[20] Xiaoling Shen and Yaoping Hou, A class of unicyclic graphs determined by their Laplacian spectrum, Electronic Journal of Linear Algebra, 23 (2012), 375-386. 